\title{
STRESS AND COPING MECHANISM OF INFERTILE WOMEN ATTENDING INFERTILITY CLINIC IN KATHMANDU
}

\author{
Sharma CK ${ }^{1}$, Subedi $D^{1}$, Rai L ${ }^{2}$, Uprety $K^{2}$
}

\begin{abstract}
INTRODUCTION: Many women face problems related to infertility in various aspects of their life. Also, it is not comfortable to discuss on this issue. Because of this, the feelings and distress faced by women may not come out and the pent up emotions may lead to different stress related problems.
\end{abstract}

MATERIAL AND METHODS : A descriptive research design was adopted to assess the stress and coping mechanism of infertile women attending infertility clinic in Kathmandu Nepal. The study was conducted from October 2011 to January 2013. Purposive sampling technique was used and sample size was 100. Data was collected through semistructured interview using; 'Perceived Stress Scale'(PSS) and 'Coping Check List' (CCL).

RESULTS: Regarding stress level of respondents, 40\% were at very high level of stress and 35\% with high level of stress. High level stress was found among the age group of 21-30 years. In relation to level of stress and coping strategies, $54.17 \%$ respondents with high stress used religious coping strategies, $50 \%$ of the respondents with very high stress also used religious coping, followed by $40 \%$ emotional coping and $37.5 \%$ problem focused coping and seeking social support strategies to overcome the stress.

CONCLUSION: It can be concluded that the almost more than half of the respondents were under high level of stress and mostly were those who are under treatment for more than 1-3 yrs of duration. The study also concluded that the higher the age the respondents were more stressed due to the infertility. It was also found that most of the respondents used religious coping as well as emotional focused coping during the stressful period.

KEYWORDS : Infertility, Infertile women, Stress, Coping mechanisms

1. Associate Professor, TUIOM, Nursing Campus, Maharajgunj, Kathmandu, Nepal

2. Lecturer, TUIOM, Nursing Campus, Maharajgunj, Kathmandu, Nepal

\author{
For Correspondence \\ Ms. Chandrakala Sharma \\ Associate Professor, \\ HOD Psychiatric Nursing, \\ Tribhuvan University, Institute of Medicine, Nursing \\ Campus, Maharajgunj \\ E-mail:csharma_s@hotmail.com
}




\section{INTRODUCTION}

The ability to bear children is very important in Nepalese Society. Most of the family member even relatives and neighbours are curious about the conception of the couple in the first year of life. Fertility is highly valued in most cultures and the wish for a child is one of the most basic of all human motivations. When attempts to have a child fail, it can be an emotionally devastating experience for the women. Infertility is not a topic which can be freely discussed in almost all cultures and communities of Nepal. Both male and female are equally responsible for the bearing of the child but most of the time society blames the female partner only. In most of the instances, people do not try to find out the reason for infertility and the versions are only relying on the saying of that the female partner is only responsible.

The incidence of infertility is gradually increasing all over the world. One estimate of overall primary and secondary infertility in South Asia, on the basis of women in their reproductive lives in the age group 45-49 years suggest an infertility rate of approximately $8-10 \%$ in India, $10 \%$ in Pakistan $11 \%$ in Srilanka, $12 \%$ in Nepal and $15 \%$ in Bangladesh (D. Kumar 2007).

Stress has been identified as a 20th century disease and has been viewed as a complex and dynamic transaction between individuals and their environments. Stressors can be broadly defined as situations or events which directly affect coping mechanism and performance of the individual. It is highly subjective so perception varies from person to person.

Coping has been viewed as a stabilizing factor that may assist individuals in maintaining psychosocial adaptation during stressful events. The process of coping is a very complex response that occurs when an individual attempts to remove stress or a perceived threat from the environment.

The criteria of infertility in this study are the women who have biological inability to conceive within a twelve months of unprotected intercourse.

\section{MATERIALAND METHODS}

Descriptive survey design, exploratory in nature, was adopted to explore the stress and different coping mechanisms of infertile women. The research sites were outpatient departments (OPD) of the Family Health Clinics of Tribhuvan University Teaching Hospital, Maharajgunj in Kathmandu. All women attending infertility clinic during the study period were the population of the study. A purposive sampling technique was adopted and the respondents were deliberately selected on the basis of criteria. The study was conducted from October 2011 to January 2013. The sample size was 100.

Structured (close ended questions), and semi structured questions were used to collect socio demographic information.

Perceived stress scale developed by Sheldon Cohen ${ }^{2,8}$ was used to assess the stress level of the respondents. The PSS is the most widely used psychological instrument for measuring the perception of stress. It is a measure of the degree to which situations in one's life are appraised as stressful. Items were designed to tap how unpredictable, uncontrollable, and overloaded respondents find their lives. The scale also includes a number of direct queries about current levels of experienced stress. There are total 10 questions related to the perceived stress scale and the criterion measures to assess the level of stress are as follows:

$12-15=$ stress free

$16-20=$ high stress

21 and above $=$ very high stress

The Perceived stress scale can be used freely, no need of permission from the author. In this study it was translated in Nepali, consistency was developed by translating in English and vice versa. It has also been used in Nepalese context by the other researcher.

\section{Coping checking list (CCL)}

This checklist was developed by Rao Subbakrishna and Prabhu (1989) ${ }^{3}$ in an Indian setting. It aims to measure the type of coping used by a person in stressful situations. It is based on a cognitive model of stress and coping.

The final version of the CCL comprises of 70 items covering a broad range of behavioral, emotional and cognitive responses that may be used in handling stressful situations. Items are scored in terms of Yes or No, indicative of the presence or absence of a particular coping behavior. Interview method was used in Nepali language to fill up the questionnaire by the researcher. This tool is already available in Nepali language in department of psychiatry and used in Nepalese context. To maintain the validity consultation with the psychiatrist, psychologists, were done. Pretesting was done in $10 \%$ of the sample size to maintain the reliability of the tool 


\section{RESULTS}

Table 1 shows the information related to marriage of the respondents. Regarding the age of marriage majority of the respondents $(49 \%)$ were married at the age of 19 to $23 \mathrm{yrs}$ whereas $19 \%$ were married at the age of $14-18$ years and $24-28$ years respectively. Regarding the duration of marriage $34 \%$ of the respondents were married for the last 2-4 years, and $23 \%$ of the respondents were married for 1-2 years and $8 \%$ of the respondents' duration of marriage was more than $14 \mathrm{yrs}$.

Table 1: Information related to marriage

\begin{tabular}{|l|l|l|}
\hline Variable & Frequency & Percent \\
\hline Age of Marriage (in yrs) & & \\
\hline $14-18$ & 19 & 19 \\
$19-23$ & 49 & 49 \\
\hline $24-28$ & 19 & 19 \\
\hline $29-33$ & 10 & 10 \\
\hline 34 and above & 3 & 3 \\
Duration of marriage (in yrs) & & \\
\hline $1-2$ & 23 & 23 \\
\hline $2-4$ & 34 & 34 \\
\hline $4-6$ & 15 & 15 \\
\hline $6-8$ & 7 & 7 \\
\hline $8-10$ & 8 & 8 \\
\hline $10-12$ & 8 & 8 \\
\hline $12-14$ & 4 & 8 \\
\hline 14 \&above & 8 & \\
\hline
\end{tabular}

Table 2: Level of stress of the respondents $n=100$

\begin{tabular}{|l|c|c|}
\hline Level of Stress & Frequency & Percent \\
\hline Stress Free (12-15) & 25 & 25 \\
\hline High Stress (16-20) & 35 & 35 \\
\hline Very High Stress & 40 & 40 \\
\hline
\end{tabular}

Table 2 reveals that majority of the respondents $40 \%$ were at very high level of stress, followed by $35 \%$ with high level of stress. Only $25 \%$ were stress free even with the problem of infertility. This finding shows that majority $75 \%$ are under stress related to infertility.

Table 3: Level of stress in relation with age of the respondents

$$
\mathrm{n}=100
$$

\begin{tabular}{|l|cc|cc|}
\hline Age (yrs) & \multicolumn{2}{|c|}{ High stress (n=35) } & \multicolumn{2}{|c|}{ Very high stress(n=40) } \\
& $\mathrm{n}$ & $\%$ & $\mathrm{n}$ & $\%$ \\
\hline Less than 21 & 1 & 2.9 & 4 & 10 \\
\hline 21 to 30 & 25 & 71.4 & 28 & 70 \\
\hline 31 to 40 & 7 & 20 & 7 & 17.5 \\
\hline Above 40 & 2 & 5.7 & 1 & 2.5 \\
\hline
\end{tabular}

Table 3 shows that out of 40 respondents who were under very high stress, $70 \%$ of them fall within the age of 21-30 yrs of age. This shows that the higher the age, the respondents were more stressed due to the infertility. It also shows that high level stress has found among the age group of 21-30 yrs of age which was $71.4 \%$ out of 35 respondents.

Table 4: Level of stress in relation to duration of treatment $\mathbf{n}=\mathbf{1 0 0}$

\begin{tabular}{|l|cc|cc|}
\hline Duration & \multicolumn{2}{|c|}{ High stress $\mathbf{( n = 3 5 )}$} & \multicolumn{2}{|c|}{ Very high stress(n=40) } \\
$\mathbf{n}$ & $\mathbf{\%}$ & $\mathrm{n}$ & $\mathbf{\%}$ \\
\hline First time & 8 & 22.9 & 6 & 15 \\
\hline Less than 1 year & 8 & 22.9 & 10 & 25 \\
\hline 1-3 yrs & 11 & 31.4 & 17 & 42.5 \\
\hline 3-6 yrs & 4 & 11.4 & 4 & 10 \\
\hline 6-9 yrs & 1 & 2.9 & 2 & 5 \\
\hline 9-12 yrs & 3 & 8.6 & 1 & 2.5 \\
\hline
\end{tabular}

Table 4 reveals findings related to relationship between treatment and stress, 11(31.4\%) out of 35 respondents who are under treatment for the duration of 1-3 yrs have high stress, followed by $22.9 \%$ under treatment for less than lyear have high stress. In relation to very high stress $17(42.5 \%)$ out of 40 respondents were under treatment for 1-3years, followed by $25 \%$ have very high stress who are undergoing treatment for less than one year.

Table 5: Major responses on coping check list $\quad n=100$

\begin{tabular}{|l|cc|}
\hline Coping Patterns * & Frequency & Percent \\
\hline Visit places of worship, go on a pilgrimage & 78 & 78 \\
\hline Talk to a family member who can do something concrete about the problem & 81 & 81 \\
You go over the problem again and again in your mind, to try to understand it & 92 & 92 \\
\hline Wish that you could change what has happened & 94 & 94 \\
\hline Analyze the problem and solve bit by bit & 94 & 94 \\
\hline Go on shopping spree & 89 & 89 \\
\hline Try to see on bright side of the things & 95 & 95 \\
\hline Talk to a friend who can do something about the problem & 91 & 91 \\
\hline Feel that time will remedy things, the only things to do are wait & 92 & 92 \\
\hline You know what has to be done so you double your efforts and try harder to & & \\
make things work. & 89 & 89 \\
\hline Seek reassurance and emotional support from family members & 71 & 71 \\
\hline Seek reassurance and support from friends & 81 & 81 \\
\hline Get away from the things for a while,take rest or vacation & 62 & 62 \\
\hline
\end{tabular}

Table 5 shows that majority $94 \%$ of the respondents used "wish that you could change what has happened and analyze the problem and solve bit by bit respectively during stress". "Talk to a family member who can do something concrete about the problem" used by $81 \%$ of the respondents. Similarly 
"You go over the problem again and again in your mind, to try to understand it" and feel that time will remedy things, the only things to do are waiting used by $92 \%$ of the respondents respectively. Majority $(95 \%)$ of the respondents tried to see the positive side of the things. "Seek reassurance and emotional support from family members" was used by $71 \%$ and "Seek reassurance and support from friends" used by $81 \%$ of the respondents. More than $60 \%$ of the respondents used to avoid the situation for awhile and go for vacation to cope with the stress.

\section{DISCUSSION}

The present study findings revealed that most of the respondents $40 \%$ were at very high level of stress, followed by $35 \%$ with high level of stress. Only $25 \%$ were stress free even with the problem of infertility. This finding shows that majority $75 \%$ were under stress related to infertility. Lukse $(1999)^{4}$ also reported similar findings that anxiety has been shown to be significantly higher in infertile couples when compared to general population, with 8 to 28 percent of infertile couples reporting clinically significant anxiety. Therefore the present study finding is supported by the literature.

This study findings reveled that majority (68\%) of the respondents visited for the first time for treatment to the government hospital followed by $14 \%$ visited traditional healers first before coming to the infertility clinic for treatment. But United Nations (1994), International Conference on Population and Development (ICPD) $)^{5}$ reported that there is inadequate focus on infertility in India's reproductive health programme. Traditionally, childless women in India experience stigma and isolation. Infertility can threaten a woman's identity, status and economic security and consequently, be a major source of anxiety leading to lowered self-esteem and a sense of powerlessness. Couples seek varied traditional methods and religious practices, including visits to temples, abstaining from visiting a place where a woman has delivered a child, observing tantric rites, wearing charms, participating in rituals and visiting astrologers.

The study findings showed that more than $60 \%$ of the respondents used to avoid the situation for awhile and go for vacation to cope with the stress. Study conducted by Nicholes et.al $(2005)^{6}$ revealed the method of coping adopted by respondents was avoidance and confrontation, avoid social gathering child. Therefore the present study finding is consistent with the literature. Same study stressed that some of the respondents wanted to solve the problems as a couple and even without children they will stay together. Similar result was found in this study as well that is majorities (94\%) of the respondents analyze the problem and solve bit by bit respectively during stress.

Communication with other people regarding infertility was not statistically associated with anxiety, with depression, with marital stress, or with marital benefit (Gourounti et al. 2012)? But in this study findings shows that the communication with friends and family was the major support available to deal with issues related to infertility. So it can be conclude that communication has a vital role to reduce stress among the infertile women in our society. More than one third of the respondents in our study mention that they used to visit places of worship, go on pilgrimages for relieving stress (religious coping strategy).

Nicholes et al. (2005) ${ }^{6}$ also reported that regarding economic consequences of infertility, most of the respondents mention that they work hard or take a loan in order to afford the treatment and are forced to suspend the treatment due to financial insufficiency that affects the couples lives and cause distress. The present study findings also revealed that $55 \%$ of the respondents had financial crisis but still they are managed by service (both self and husband) and also by the family members.

The present study findings revealed that $55 \%$ of the respondents faced financial problems related to infertility, followed by $32 \%$ of the respondents had low self esteem and $25 \%$ had loss of confidence in day to day activities, and $20 \%$ had marital stress in relation with infertility. Similar result was found by Zuraida AS in 2010 in a study on Psychological Distress Among Infertile Women reported that the experience of infertility is linked with psychological responses such as depression, anxiety, guilt, social isolation, and decreased selfesteem in both men and women. Therefore the above literature supported the present study findings.

\section{CONCLUSION}

It can be concluded that the almost more than half of the respondents were under high level of stress and mostly were those who are under treatment for more than 1-3 yrs of duration. The study also concluded that the higher the age the respondents were more stressed due to the infertility. It was also found that most of the respondents used religious coping as well as emotional focused coping during the stressful period. It would be recommended that the health personnel need to counsel those who visit the infertility clinic and 
provide support to those under stress.

\section{ACKNOWLEDGEMENT}

We would like to express gratitude to University Grant Commission, Sanothimi, Kathmandu, Nepal for providing fund to conduct this study.

\section{REFERENCES}

1. Prasanta Kumar D, Sharma. Psychological aspects of infertility, BJMP, 2010;3(3)-a336

2. http://www.stress-relief-tools.com/symptoms-ofstress. html retrived on 2011-07-08.

3. Rao K, Subbakrishna D.K, \&Prabhu, G.G. Development of Coping Check list-A Preliminary Report. Indian Journal of Psychiatry1989;31,2,128-133.

4. Lukse MP, Vacc NA. Grief, depression, and coping in women undergoing infertility treatment.Obstet and Gynecol 1999;93 (2):245-251.

5. Fertility and Mental Health $\mid$ MGH Center for Women's Mental Health 2005

6. Nicholes W,\& et.al. Consequences of infertility in developing countries in South of Vietnam, Journal of translational medicine, 2005; $4: 54$

7. Gourount k,Lykeridou K,Vaslamatzis G. Increased anxiety and depression in Greek infertile women results from feelings of marital stress and poor marital communication. Health Science Journal, 2012; Volume 6, Issue 1 (January March 2012).

8. Cohen, S; Kamarck T; Mermelstein R. A global measure of perceived stress. Journal of Health and Social Behavior, December 1983; 24 (4): 385396.

doi:10.2307/2136404. PMID 6668417. 